\title{
Comportamento e características de usuários de lentes de contato entre estudantes universitários da área de saúde
}

\author{
The behavior and characteristics of contact lens wearers among university students \\ in health related areas
}

Cleusa Coral Ghanem ${ }^{(1)}$

Ramon Coral Ghanem (2)

Geann Wellington De Bortoli (2)

Ester Sakae Yamazaki ${ }^{(2)}$

\begin{tabular}{|l} 
RESUMO \\
\hline Objetivo: Estudar o comportamento e características de usuários \\
de lentes de contato ligados à área de saúde. \\
Método: Realizou-se um levantamento entre universitários da área \\
de saúde da Pontifícia Universidade Católica do Paraná, em Curitiba, \\
no período de 23 a 27 de novembro de 1998 . Para a coleta de dados foi \\
utilizado um questionário auto-aplicável, previamente testado. \\
Resultados $e$ Discussão: Dentre 1.173 estudantes, 207 (17,2\%) \\
usavam lentes de contato, sendo 78,3\% do sexo feminino e $21,7 \%$ do \\
sexo masculino. Eram usuários de lentes rígidas $12,1 \%$ e $87,9 \%$ \\
utilizavam hidrofílicas, demonstrando um aumento progressivo das \\
últimas quando se compara a estudos nacionais anteriores. Dos \\
usuários de lentes de contato hidrofílicas, $57,6 \%$ utilizavam descar- \\
táveis e desses $88,5 \%$ não as utilizavam de acordo com os padrões de \\
uso e descartabilidade estabelecidos. Do número total de usuários, \\
$37,7 \%$ relataram algum problema ocular durante o uso. Embora \\
97,1\% tenham feito a adaptação com o oftalmologista, $14,2 \%$ dos \\
usuários de descartáveis não obtiveram novas lentes em clínicas \\
oftalmológicas. Por outro lado, $81,1 \%$ procuraram o oftalmologista, \\
semestral ou anualmente, para a revisão da adaptação. \\
Recomendação: Para reduzir o número de complicações a desistên- \\
cia do uso de lentes de contato, o usuário deve ser educado sobre a forma \\
correta de utilizá-las, ser orientado sobre os sinais e sintomas de alerta \\
para problemas oculares e receber as informações também por escrito.
\end{tabular}

Palavras-chave: Lentes de contato; Lentes de contato descartáveis; Comportamento de usuários de lentes de contato.

\section{INTRODUÇÃO}

Segundo resultados de pesquisa realizada em 1995 por Coral-Ghanem ${ }^{1}$, na presidência da Sociedade Brasileira de Lentes de Contato e Córnea (SOBLEC), havia no Brasil, aproximadamente, 1.700.000 usuários de lentes de contato (LC). Desse total, cerca de $46 \%$ usavam lentes hidrofílicas esféricas de uso diário, $16 \%$ hidrofílicas esféricas de uso prolongado, $5 \%$ descartáveis, $25 \%$ rígidas gás-permeáveis e $7 \%$ outros tipos.

O desenvolvimento de novos materiais e desenhos, fácil acesso aos fabricantes estrangeiros, desenvolvimento de soluções multi-uso, diminuição do custo e popularização das LC, têm aumentado o número de adaptações, principalmente de descartáveis, modificando o comportamento e as características dos usuários.
Os autores declaram que não possuem interesse financeiro nos resultados desse estudo.

Médica Oftalmologista - Chefe do Setor de Lentes de Contato do Hospital de Olhos Sadalla Amin Ghanem.

Doutorandos do Curso de Medicina da Universidade Católica do Paraná.

Endereço para correspondência: Dra. Cleusa Coral Ghanem. Hospital de Olhos Sadalla Amin Ghanem

- R. Abdon Batista, 172 - Centro - Joinville (SC) CEP 89201-010. Email: coral@ netville.com.br 
As LC descartáveis, lançadas nos Estados Unidos da América em 1987, foram desenvolvidas para reduzir a necessidade de manuseio e manutenção, a contaminação da superfície, a probabilidade de causar micro-trauma corneano por inserção e remoção repetidas, as complicações secundárias a depósitos e também para evitar complicações relacionadas a sensibilidade às soluções de manutenção ${ }^{2}$.

A orientação quanto ao tempo de uso e descartabilidade, aprovada pelo FDA (The Food and Drug Administration) e recomendada pela SOBLEC, ratificada por Holzchuh ${ }^{3}$ é a seguinte: uso contínuo de até 6 noites e 7 dias, descansando uma noite, e no dia seguinte colocando um novo par de lentes; ou uso diário, descartando em 14 dias.

O uso contínuo com descartabilidade semanal diminuiu o número de complicações de modo geral, entretanto, com freqüência provoca ceratite puntada superficial ${ }^{4} \mathrm{e}$ edema corneano, aumentando o risco de úlcera de córnea, que representa a mais temida das complicações ${ }^{5}$. Assim, é preferível recomendar o uso diário.

As LC descartáveis foram lançadas no mercado brasileiro acompanhadas por intensa propaganda de suas vantagens e pouco esclarecimento sobre suas limitações. Houve, por parte de muitos profissionais, uma falta de entendimento quanto à nova filosofia de adaptação, o que provocou diferentes orientações quanto ao esquema de uso e descartabilidade a ser repassado aos usuários. Além disso, o uso incorreto das orientações recebidas por parte desses usuários, tem provocado desistência do uso de LC e complicações oculares facilmente evitáveis.

O objetivo deste estudo é, portanto, verificar o comportamento de usuários de LC que, por estarem ligados à área da saúde, supõe-se serem mais conscientes a respeito da preservação da saúde e importantes agentes responsáveis pela divulgação de informações sobre o uso correto das LC à população em geral. Além disso, como alunos de uma universidade particular, representam uma classe que, provavelmente, apresenta menos problemas financeiros, o que facilita a troca das lentes no tempo determinado, a utilização dos métodos adequados de manutenção e o controle oftalmológico periódico. Devem fazer parte, a princípio, da parcela da população que alcança melhores resultados no uso de LC. Nesse sentido, constituíram os objetivos específicos deste trabalho verificar:

1 - a proporção de usuários de LC;

2 - as características pessoais dos usuários;

3 - os tipos de LC utilizados;

4 - a conduta dos usuários de LC rígidas e hidrofílicas;

5 - os problemas relatados pelos usuários;

6 - a freqüência do controle de adaptação.

\section{MATERIAIS E MÉTODOS}

Realizou-se um levantamento entre estudantes universitários da área de saúde da Pontifícia Universidade Católica do Paraná (PUC-PR), em Curitiba no período de 23 a 27 de novembro de 1998. Para a coleta de foi utilizado um questionário auto-aplicável, previamente testado. Integraram a amostra alunos das turmas do $1^{\circ}$ ao $4^{\circ}$ ano dos cursos de farmácia, fisioterapia, fonoaudiologia, medicina, odontologia e psicologia presentes na sala de aula naquele período e que aceitaram responder ao questionário. Para conhecer a proporção de usuários de LC naquela população universitária, foi solicitado aos estudantes que preenchessem o instrumento de acordo com o seu caso (usuário ou não de LC). Posteriormente, os não-usuários foram separados, de forma que a amostra foi composta apenas por usuários de LC. O tempo médio para completar o questionário foi de quinze minutos, com devolução imediata aos pesquisadores.

\section{RESULTADOS}

1. Proporção de usuários de LC - Responderam o questionário 1173 pessoas, das quais 207 (17,6\%) usavam LC (figura 1).

2. Características pessoais - As características pessoais dos usuários encontram-se na tabela 1.

3. Tipos de LC utilizadas - Dos 207 usuários, 25 (12,1\%) usavam LC rígidas e 182 (87,9\%), LC hidrofílicas (LCH).

4. Conduta dos usuários de LC rígidas e hidrofílicas: O tempo médio de uso das LC rígidas foi 5,2 anos e das $\mathrm{LCH}$ foi 2,6 anos. A forma de uso diário foi a mais freqüente (tabela 2). A maioria dos usuários $(97,1 \%)$ adaptou suas LC em consultório oftalmológico, embora cerca de $10 \%$ não obtém novas LC nesse local (tabela 3).

Dentre os usuários de LCH, 105 (57,7\%) utilizavam LCH descartáveis e 77 (42,3\%), LCH convencionais. Quanto a forma de uso das LCH descartáveis, o uso diário com descarte mensal foi o mais comum $(25,7 \%)$, seguido pelo uso diário por mais de um mês (21\%) (tabela 4$)$.

5. Problemas relacionados ao uso de LC - Tiveram algum problema 78 usuários $(37,7 \%)$. Para a descrição dos problemas relatados ver tabela 5 .

6. Freqüência do controle de adaptação - Mostrou ser semestral para $43 \%$ e anual para $38 \%$ (figura 2 ).

\section{DISCUSSÃO}

O número de usuários de LC, em valores absolutos, foi

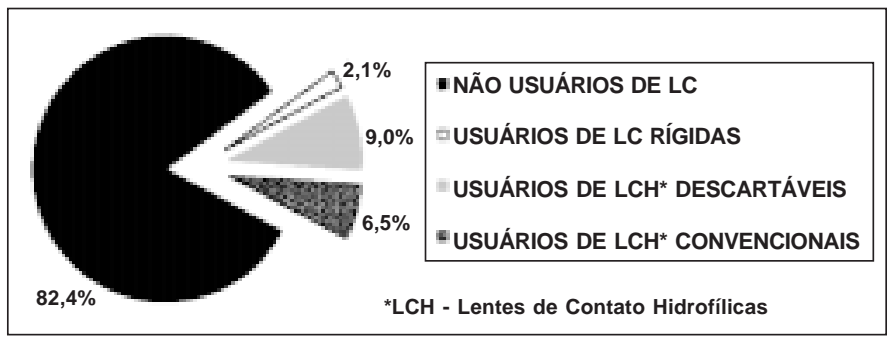

Fig. 1 - Proporção de usuários de lentes de contato, segundo o tipo de contato $(n=1173)$. 


\begin{tabular}{|c|c|c|}
\hline \multicolumn{3}{|l|}{ Características } \\
\hline IDADE (anos) & \multicolumn{2}{|c|}{$20,2 \pm 2,3$} \\
\hline SEXO & f & $\%$ \\
\hline Masculino & 45 & 21,7 \\
\hline Feminino & 162 & 78,3 \\
\hline \multicolumn{3}{|l|}{ COR DA PELE } \\
\hline Branco & 194 & 93,7 \\
\hline Negro & 8 & 3,9 \\
\hline Amarelo & 5 & 2,4 \\
\hline \multicolumn{3}{|c|}{ CURSO FREQÜENTADO } \\
\hline Medicina & 50 & 24,2 \\
\hline Fisioterapia & 45 & 21,8 \\
\hline Psicologia & 44 & 21,3 \\
\hline Odontologia & 40 & 19,3 \\
\hline Farmácia & 16 & 7,7 \\
\hline Fonoaudiologia & 12 & 5,7 \\
\hline
\end{tabular}

\begin{tabular}{|c|c|c|c|c|}
\hline \multirow[t]{3}{*}{ Forma de uso } & \multicolumn{4}{|c|}{ Tipo de lentes de contato } \\
\hline & \multicolumn{2}{|c|}{ Hidrofílicas $(n=182)$} & \multicolumn{2}{|c|}{ Rígidas $(n=25)$} \\
\hline & f & $\%$ & $f$ & $\%$ \\
\hline Diário & 112 & 61,5 & 19 & 76,0 \\
\hline Contínuo / Flexível & 40 & 22,0 & 3 & 12,0 \\
\hline Ocasional & 30 & 16,5 & 3 & 12,0 \\
\hline
\end{tabular}

significativamente maior no sexo feminino do que no masculino. Entretanto, considerando-se a superioridade do número de mulheres que estudam no setor de ciências biológicas e da saúde da PUC-PR, e que participaram do estudo, proporcionalmente, encontrou-se que $18,4 \%$ de mulheres e $15,2 \%$ de homens usavam LC; o que não representa uma grande diferença de interesse pelo uso de LC entre os sexos. Isso tem demonstrado um maior interesse dos homens pelas LC, justificado não somente pelo aumento da vaidade masculina, observada nas outras áreas de estética, como também pela importância da aparência na busca de emprego, muitas vezes prejudicada pelo uso de óculos.
Quanto aos tipos de LC, 12,1\% usavam rígidas, enquanto em 1997 a percentagem numa população semelhante, era $15 \%{ }^{6}$ e, em 1995, conforme pesquisa da SOBLEC ${ }^{1}$, era $25 \%$. Apesar dos grandes benefícios das LC rígidas gás-permeáveis (RGP) no que se refere à qualidade de visão, à maior facilidade de oxigenação corneana quando comparada à lente hidrofílica, à facilidade de manutenção, o número de adaptações das RGP está diminuindo progressivamente. As causas mais prováveis são o desenvolvimento de melhores LCH tóricas, além do interesse progressivo pela cirurgia refrativa, especialmente entre usuários insatisfeitos com o conforto da LC rígida.

$\mathrm{O}$ uso de LCH representa, neste estudo, a maioria dos usuários $(87,9 \%)$ e dentre eles $57,7 \%$ utilizam as descartáveis. $\mathrm{O}$ aumento do uso das hidrofílicas pode ser explicado, principalmente, pelo desenvolvimento das lentes descartáveis, das hidrofílicas para a correção do astigmatismo e das soluções multi-uso, que têm aumentado a praticidade de manutenção.

Em 1997, Kara-José e cols. ${ }^{6}$ encontraram, dentre 80 usuários de LC, $85 \%$ hidrofílicas, das quais $42,5 \%$ eram descartáveis. Quando se compara esse número com o percentual en-contrado nesta pesquisa $(57,7 \%)$, percebe-se um crescimento significativo de uso de descartáveis para o período. Este crescimento é mais evidente quando se compara com o número relatado pela pesquisa nacional feita pela SOBLEC ${ }^{1}$, em 1995, quando apenas 5\% do total de usuários utilizava LC descartáveis e reflete a tendência atual de exigência de conforto e praticidade. Esse aumento, porém, tem sido acompanhado do uso indiscriminado que provoca complicações oculares facilmente evitáveis.

Quanto a forma de uso das LC descartáveis, 6 pessoas $(5,7 \%)$ usavam continuamente por 15 dias, $54(51,4 \%)$ descartavam as LC com 1 mês de uso, 33 (31,5\%) com mais de 1 mês. Ou seja, $88,6 \%$ dos usuários da amostra faziam uso incorreto das LC descartáveis. Outro estudo ${ }^{7}$, demonstrou em um grupo de 145 pacientes usuários de descartáveis, que $69 \%$ procediam incorretamente quanto ao tempo de uso e substituição das lentes. Ainda a respeito do uso das descartáveis, $33(31,4 \%)$ faziam uso contínuo e desses 31 (93,9\%) não obedeciam o tempo de descartabilidade. Nesse grupo, 15 (45\%) relataram problemas durante o uso. A principal razão parece ser a não obediência dos usuários quanto ao tempo de uso e descartabi-

\begin{tabular}{|c|c|c|c|c|c|c|}
\hline \multirow[t]{3}{*}{ Local } & \multirow{2}{*}{\multicolumn{2}{|c|}{$\begin{array}{l}\text { Rígidas } \\
(\mathrm{n}=25)\end{array}$}} & \multicolumn{4}{|c|}{ Hidrofílicas } \\
\hline & & & \multicolumn{2}{|c|}{$\begin{array}{c}\text { Descartáveis } \\
(n=105)\end{array}$} & \multicolumn{2}{|c|}{$\begin{array}{c}\text { Convencionais } \\
(n=77)\end{array}$} \\
\hline & f & $\%$ & f & $\%$ & f & $\%$ \\
\hline \multicolumn{7}{|l|}{ PARA ADAPTAÇÃO } \\
\hline Consultório Oftalmológico & 25 & 100 & 101 & 96,2 & 75 & 97,4 \\
\hline Casa de Ótica & - & - & 4 & 3,8 & 2 & 2,6 \\
\hline \multicolumn{7}{|l|}{ PARA OBTENÇÃO } \\
\hline Consultório Oftalmológico & 25 & 100 & 90 & 85,8 & 71 & 92,2 \\
\hline Casa de Ótica & - & - & 12 & 11,4 & 6 & 7,8 \\
\hline Mala direta & - & - & 2 & 1,9 & - & - \\
\hline
\end{tabular}




\begin{tabular}{|c|c|c|c|c|c|c|c|c|}
\hline \multirow[t]{3}{*}{ Forma de uso } & \multicolumn{8}{|c|}{ Descarte das lentes } \\
\hline & \multicolumn{2}{|c|}{ Semanal } & \multicolumn{2}{|c|}{ Quinzenal } & \multicolumn{2}{|c|}{ Mensal } & \multicolumn{2}{|c|}{ Depois de um mês } \\
\hline & f & $\%$ & f & $\%$ & f & $\%$ & f & $\%$ \\
\hline Diário & 2 & 1,9 & 6 & 5,7 & 27 & 25,7 & 22 & 21,0 \\
\hline Contínuo / Flexível & 2 & 1,9 & 6 & 5,7 & 18 & 17,1 & 7 & 6,7 \\
\hline Ocasional & 1 & 0,9 & 1 & 0,9 & 9 & 8,6 & 4 & 3,8 \\
\hline
\end{tabular}

\begin{tabular}{|c|c|c|c|c|c|}
\hline $\begin{array}{l}\text { Problemas com Rígidas } \\
(n=15)\end{array}$ & f & $\%$ & $\begin{array}{l}\text { Problemas com Hidrofílicas } \\
(n=63)\end{array}$ & f & $\%$ \\
\hline Dificuldade de adaptação & 6 & 40,0 & Irritação & 20 & 31,8 \\
\hline Conjuntivite & 4 & 26,6 & Conjuntivite & 13 & 20,6 \\
\hline Irritação & 3 & 20,0 & Alergia & 8 & 12,7 \\
\hline Problemas de manuseio & 1 & 6,7 & Depósitos e contaminações & 6 & 9,5 \\
\hline \multirow[t]{4}{*}{ Lesão de córnea } & 1 & 6,7 & Ceratite & 5 & 7,9 \\
\hline & & & Úlcera de córnea & 4 & 6,4 \\
\hline & & & $\mathrm{CPG}^{*}$ & 2 & 3,2 \\
\hline & & & Outros & 5 & 7,9 \\
\hline
\end{tabular}

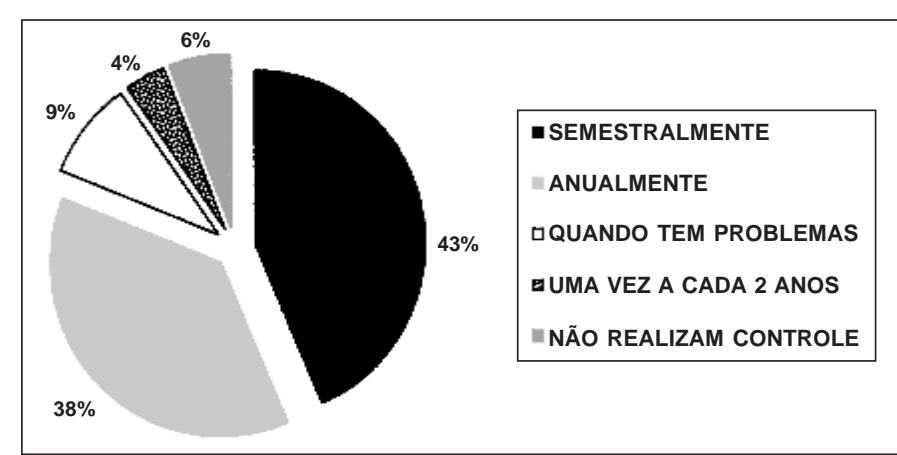

Fig. 2 - Frequência do controle de adaptação das lentes de contato ( $n=207)$.

lidade, às vezes decorrente da facilidade de aquisição das LC em estabelecimentos comerciais ${ }^{8}$, por venda direta ao usuário, sem supervisão e orientação médica. O uso contínuo aumenta o número de complicações de modo geral ${ }^{9}$, e aumenta de 10 a 15 vezes o risco de ceratite infecciosa comparando-se ao uso diario ${ }^{10,11}$. A hipóxia, alterações do epitélio, e a presença de bactérias na LC formam a tríade causadora do desenvolvimento de úlcera corneana infecciosa ${ }^{10,11}$. Um estudo retrospectivo de Alves ${ }^{12}$ demonstrou que as LC descartáveis em regime de troca planejada, sob supervisão médica, são uma boa alternativa para pacientes que não tiveram sucesso com outros tipos de LC.

Embora a maioria $(97,1 \%)$ das pessoas pertencentes a este estudo tenha adaptado suas LC com o oftalmologista, 14,2\% dos usuários de descartáveis não obtém novas lentes em clínicas oftalmológicas, levando a uma quebra do vínculo médicopaciente. Somado-se a isso, verificou-se a falta de obediência à regra básica de troca das LC, importante fator para manter a saúde ocular.

Esses resultados reforçam a necessidade do oftalmologista - em seu consultório - continuar orientando o usuário, verbal e por escrito, em relação ao uso correto das LC. O usuário deverá ser conscientizado de que a adaptação termina somente quando deixar de usá-las e que esse processo dinâmico representa um equilíbrio frágil entre o olho e a LC, que pode ser rompido a qualquer momento por uma série de fatores.

Os oftalmologistas têm a responsabilidade de fornecer acompanhamento apropriado aos pacientes. Manter um arquivo separado dos usuários de LC descartáveis é uma valiosa estratégia para identificar pacientes que não retornam para controle oftalmológico, colocando em risco sua saúde ocular.

\section{SUMMARY}

Purpose: To study the behavior and characteristics of contact lens wearers involved in health areas.

Methods: To conduct a survey among students of health related courses at the Pontifícia Universidade Católica do Paraná, in Curitiba, during the period from $23^{\text {rd }}$ to $27^{\text {th }}$ November 1998. A previously tested, self-applicable questionnaire was used to collect data.

Results and Discussion: Among 1,173 students, 207 (17.2\%) used contact lenses, of which $78.3 \%$ were female and $21.7 \%$ were male. Twelve point one percent (12.1\%) used rigid and $87.9 \%$ soft lenses, showing a progressive increase in the use of soft lenses when compared to previous national studies. Of the 
soft lens wearers, $57.6 \%$ used disposable lenses and of these, $88.5 \%$ did not follow the established standards for use and disposability. Of the total number of wearers, $37.7 \%$ reported ocular problems during contact lens use. Although $97.1 \%$ of the individuals of this study were fitted for contact lenses by an ophthalmologist, $14.2 \%$ obtained new lenses outside ophthalmologic clinics. On the other hand, $81 \%$ consulted an ophthalmologist one or twice yearly to check lens fitting.

Recommendation: To reduce the number of complications and the discontinuance of wearing lenses, the user must be properly educated in the use of lenses and how to identify signs and symptoms indicating ocular problems as well as having this information in writing.

Keywords: Contact lenses, Disposable contact lens, Behavior of contact lens wearers.

\section{REFERÊNCIAS BIBLIOGRÁFICAS}

1. Coral-Ghanem C. Pesquisa SOBLEC; Jornal da Sociedade Brasileira de Lentes de Contato e Córnea - SOBLEC 1995;4.
2. Gruber E. The Disposable Contact Lens: A New Concept in Extended Wear. The CLAO Journal 1998:14(4):195-8.

3. Holzchuh N. Lentes descartáveis e de troca planejada. In: Coral-Ghanem C, Kara-José N. Lentes de Contato na clínica Oftalmológica. Ed. Cultura Médica 1998;8:49-51.

4. Watanabe K, Hamano H. The Typical Pattern of Superficial Punctate Keratopathy in Wearers of Extended Wear Disposable Contact Lenses, The CLAO Journal 1997;23(2):134-7.

5. Poggio EC, Glynn RJ, Schein OD et al. The incidence of ulcerative keratitis among users of daily-wear and extended wear soft contact lenses. N Engl J Med 1989;321:779-83.

6. Kara-José $\mathrm{N}$ e cols. Perfil dos usuários de lentes de contato em profissionais da área de saúde. Rev Med São Paulo 1997;76(6):307-14.

7. Smith SK. Patient noncompliance with wearing and replacement schedules of disposable contact lenses. J Am Optom Assoc 1996;67(3):160-4.

8. Maia EM, Pietrovicz J, Maia M, Ramos ARB, Moreira JR. CA. Adaptação e venda de lentes de contato pelas ópticas de Curitiba. Revista Brasileira de Oftalmologia 1999;58(5):396-400.

9. Suchecki JK, Ehlers WH, Donshik PC. Peripheral Corneal Infiltrates Associated With Contact Lens Wear. The CLAO Journal 1996;22(1):41-6.

10. Coral-Ghanem C, Kara-José N. Complicações das Lentes de Contato. In Coral-Ghanem C, Kara-José N. Lentes de Contato na clínica Oftalmológica. Ed. Cultura Médica 1998;19:133-6.

11. Weissman BA, Donzis PB, Hoft RH. Keratitis and Contact Lens Wear: a review. J Am Optom Assoc 1987;58(10):799-803.

12. Alves MR. Avaliação clínica de lentes de contato descartáveis em regime de troca planejada em usuários de lentes NewVues e Focus em uso diário. Rev Bras de Oftal 1998;57(9):703-9.

\title{
$4^{\circ}$ Congresso da Associação Centro-Oeste de Oftalmologia
}

\author{
22 a 24 de Junho de 2000 \\ Cuiabá - Mato Grosso
}

INFORMAÇÕES: RKF Publicidade/ K2 Assessoria e Eventos

Av. Getulio Vargas, 1397 - Sala 104

Ed. Avenida Central - Centro - Cuiabá - MT

Fone: (0xx65) 624-3454 